\title{
Ein "Irrthum und die Möglichkeit, ihn selbst öffentlich zurückzunehmen" Jherings Lösungsansatz zu Paul. D. 18.4.21 (Gefahrtragung beim Doppelverkauf)
}

\section{1. "Anfangsworte"}

Im Jahr 2007 wurde in einem Wettbewerb der "Initiative Deutsche Sprache" und der "Stiftung Lesen" der schönste erste Satz in der deutschsprachigen Literatur gekürt ${ }^{1}$. Gäbe es ein vergleichbares Ranking für Anfangsworte in wissenschaftlichen Arbeiten, so hätte ein Beitrag von Rudolf von Jhering zum Gefahrtragungsproblem im "Fall des mehrfachen Verkaufs" wohl gute Chancen auf einen der vorderen Plätze²:

"Die Beschäftigung mit dem Gegenstand der vorliegenden Abhandlung ist mir in doppelter Weise recht lehrreich gewesen, sie hat mich zwei auf die Erkenntniß des Rechts bezügliche Erfahrungen machen lassen. Die erste: daß es sich mit der Erkenntniß der wahren Natur der Rechtssätze ähnlich verhält, wie mit der der Menschen, insofern es nämlich bei beiden oft einer ganz besondern Lage, einer ungewöhnlichen, sie auf die Probe stellenden Situation bedarf, um ihr wahres Wesen zum Vorschein zu bringen".

Während allerdings die so beschriebene "erste Erfahrung" von manchem unter Hinweis auf den "Erfahrungssatz ... that hard cases make bad law" eher bezweifelt wird ${ }^{3}$, erweckt die oben bereits angedeutete "zweite"4 gerade im Bezug auf Methodenfragen größtes Interesse

1 Die Wahl der "prominent besetzten Jury" (s. spiegel online: http://www.spiegel.de/kultur/literatur/ schoenster-romananfang-gekuert-der-erste-satz-geht-an-grass-a-515792.html) fiel auf den Roman "Der Butt" (1977) von Nobelpreisträger Günter Grass: "Ilsebill salzte nach". Im internationalen Vergleich rittern etwa Leo Tolstoj (Anna Karenina), Jane Austen (Stolz und Vorurteil) oder Charles Dickens (Eine Geschichte aus zwei Städten) um die Gunst, als schönster Romananfang zu gelten; vgl. als ein Beispiel aus der Literatur zum Thema Harald Beck, Roman-Anfänge, Zürich 1992, 129 f.

2 Rudolf v. Jhering, Beiträge zur Lehre von der Gefahr beim Kaufcontract. I. Ueber den Sinn des Satzes: Der Käufer trägt die Gefahr, mit besonderer Beziehung auf den Fall des mehrfachen Verkaufs, in: JherJB 3 (1859), 449-488, 449 [= ders., Gesammelte Aufsätze aus den Jahrbüchern für die Dogmatik des heutigen römischen und deutschen Privatrechts, Bd. 1, Jena 1881 (Ndr. Aalen 1969), 291-326].

3 Vgl. Ulrich Falk, Ein Gelehrter wie Windscheid. Erkundungen auf den Feldern der sogenannten Begriffsjurisprudenz, Frankfurt/Main 1989, 70.

$4 \quad$ Jhering, Sinn (Fn. 2), 450: "... damit bezeichne ich meine zweite Erfahrung ... Es ist in der That ein anderes Ding, unbekümmert um die Folgen und das Unheil, das ein Rechtssatz, den man in den Quellen zu lesen oder aus der Consequenz zu entnehmen glaubt, im Leben anstiftet, sich rein theoretisch mit ihm 


\section{PENNITZ}

in der neueren Jhering-Forschung ${ }^{5}$ : Denn inwiefern dürfe man im Hinweis auf das "Unheil", das "aus der Consequenz" resultieren kann, tatsächlich - nach dem oft zitierten Diktum Wieackers ${ }^{6}$ - Jherings "Damaskuserlebnis" oder eben "den ersten öffentlichen Schritt in die neue Bahn", also eine Abkehr von der "Begriffsjurisprudenz" eines Puchta sehen, wie es in "Scherz und Ernst" vom Autor selbst rückblickend festgehalten wird7.

Jedenfalls war Jhering in den Jahren 1858/59 "praktisch an einem wirklichen Rechtsfall" mit der heiklen Frage der Gefahrtragung beim Doppelverkauf konfrontiert ${ }^{8}$ : Ein englisches Handelshaus hatte einem anderen einige Schiffsparten ${ }^{9}$ zum Kauf angeboten. Als die Verhandlungen (vorerst) zu keinem Abschluss führten, wurde eine Parte daraus an einen Rostocker Unternehmer verkauft und später sämtliche Parten - im Vertrauen darauf, den Erstverkauf rückgängig machen zu können - an den ursprünglichen Interessenten veräußert. Allerdings kam es im weiteren Verlauf nicht mehr zur Rückabwicklung des ersten Vertrags, da das betreffende Schiff in der Zwischenzeit untergegangen war. Angesichts der Tatsache, dass die Gefahr gegenüber dem Zweitkäufer - nach damaligem englischen Recht - beim Verkäufer lag, verlangte letzterer nun unter Berufung auf die periculum emptoris-Regel des gemeinen Rechts den vereinbarten Kaufpreis vom Erstkäufer. Hätte man etwa beide Vertragsabschlüsse nach römisch(-gemein)em Recht zu beurteilen gehabt, so wäre aus diesem Rechtssatz eventuell sogar abzuleiten, dem Verkäufer sei angesichts des zufälligen Sachuntergangs der Preis zwei Mal zuzusprechen: Genau zu diesen "Resultaten ..., gegen die das natürliche Rechtsgefühl Protest erhebt"10 war Jhering nämlich - gestützt auf Paul.

abzufinden oder aber ihn zur Anwendung zu bringen. Eine ungesunde Ansicht, wenn sonst nur das Subject selbst noch gesund ist, hält eine solche Probe nicht aus".

5 Vgl. nur Okko Behrends, Das 'Rechtsgefühl' in der historisch-kritischen Rechtstheorie des späten Jhering, in: ders. (Hg.), Rudolf von Jhering. Über die Entstehung des Rechtsgefühls, Napoli 1986, 55-184, 71 u. Fn. 17; 72-75; ders., Rudoph von Jhering (1818-1892). Der Durchbruch zum Zweck des Rechts, in: F. Loos (Hg.), Rechtswissenschaft in Göttingen, Göttingen 1987, 229-269, 246 ff.; 252 ff., bes. 253 f. u. Fn. 78; Falk, Gelehrter (Fn. 3), 10-15, bes. 10 u. Fn. 55 bzw. 12 u. Fn. 65 f.; 52-72, bes. 56; 65; 70; Michael Kunze, Rudolf von Jhering - ein Lebensbild, in: O. Behrends (Hg.), Rudolf von Jhering. Beiträge und Zeugnisse, 2. Aufl., Göttingen 1993, 11-28, 16 f.; oder zuletzt Ilse Kroppenberg, Die Plastik des Rechts. Sammlung und System bei Rudolf v. Jhering, Berlin 2015, 21-34, bes. 30 ff. u. Fn. 96 bzw. Ralf Seinecke, Methode und Zivilrecht beim "Begriffsjuristen" Jhering (1818-1892), in: J. Rückert/ders. (Hg.), Methodik des Zivilrechts - von Savginy bis Teubner, 3. Aufl., Baden-Baden 2017, 148-176, $168 \mathrm{f.}$ (Rn. 410/6); 171 (Rn. 416 f.).

$6 \quad$ Franz Wieacker, Privatrechtsgeschichte der Neuzeit, 2. Aufl., Göttingen 1967, 451, freilich mit dem schon auf die "Jahrbücher für Dogmatik" bezogenen Zusatz (ders., Gründer und Bewahrer, Göttingen 1959, 203 f.): "Bei näherem Zusehen verlassen schon diese Schriften den traditionellen Boden der historischen Schule"; s. dazu auch ders., Rudolph von Jhering, in: ZRG RA 86 (1969), 1-36, bes. 22 u. Fn. 92; 23-25 u. Fn. 94; zum "Damaskuserlebnis" etwa - um ein Beispiel zu nennen - Seinecke, Methode (Fn. 5), 169 (Rn. 411).

$7 \quad$ Rudolf v. Jhering, Scherz und Ernst in der Jurisprudenz, Leipzig 1884, 337-339 u. Fn. 1.

8 Jhering, Sinn (Fn. 2), 450; $451 \mathrm{f}$.

9 Zu dem in der neueren Literatur z. T. als Anteil an einer Schiffsladung (z. B. Kohle) missverstandenen Begriff, der vielmehr den ideellen Gesellschafts-/Eigentumsanteil "an einer Partenreederei zum Betrieb eines Hochseeschiffs" meint, nur Kroppenberg, Plastik (Fn. 5), 21 f. u. Fn. 60/62 bzw. Seinecke, Methode (Fn. 5), 169 Fn. 57.

10 Jhering, Sinn (Fn. 2), 450. 
D. 18.4.21 - in einer früheren Abhandlung gelangt, doch schwingt dabei schon 1844 ein gewisses Unbehagen mit $^{11}$.

Im Folgenden soll Jherings "brillianter" Beitrag zur Gefahrtragung aus $1859^{12}$ freilich nicht Anlass geben, dem großen und vieldiskutierten Thema seiner zivilrechtlichen Methode Weiteres hinzuzufügen, das Interesse gilt vielmehr einem etwas in den Hintergrund getretenen Aspekt. Denn obwohl es Jhering vor allem darum ging, den vorliegenden Rechtsfall im Wege "restrictiver Interpretation" des römischen periculum emptoris-Satzes gerecht zu lösen ${ }^{13}$ und der Rechtsfigur darüber hinaus grundsätzlich eine der damaligen "Zeitstufe" adäquate Gestalt zu geben ${ }^{14}$, hatte er sich hierzu doch in einem ersten Schritt "als Romanist"15 dem bereits angesprochenen Paulusbeleg zuzuwenden: Insofern soll an dieser Stelle kurz überlegt werden, was die heutige römisch-rechtliche Lehre Jherings Aufsatz schuldet bzw. verdanken könnte.

\section{Jhering als wegweisender Romanist}

Jhering bezieht sich in seiner Argumentation auf folgenden Exkurs aus der deutlich längeren Entscheidung Paul. (16 quaest. ${ }^{16}$ ) D. 18.4.21, die von einem Erbschaftsverkauf ausgeht:

11 Vgl. Rudolph Jhering, In wie weit muß der, welcher eine Sache zu leisten hat, den mit ihr gemachten Gewinn herausgeben?, in: ders., Abhandlungen aus dem Römischen Recht, Leipzig 1844, 1-86, 59: "Wenn der Verkäufer aus eigennütziger Absicht die verkaufte Sache noch einmal veräußert, so braucht er, selbst wenn die Sache durch casus untergegangen ist, der Käufer also den Kaufpreis zahlen muß, ohne die Sache zu bekommen, dennoch den vom zweiten Käufer erhaltenen Preis nicht zu restituiren"; dazu dann ebd., 71: "Hätte man statt juristischer Consequenz Milde eintreten lassen wollen, so wäre sie weit eher in dem Falle der 1. 21 de her. vend. ... begründet und nöthig gewesen", und abschließend (ebd., 86): "Hätten alle römischen Juristen die rigoristische Consequenz des Paulus getheilt, so würde ich den Vorwurf nicht fürchten, auf den ich jetzt allerdings gefasst bin: meinem Princip zu Liebe Consequenzen zugegeben zu haben, zu denen sich die römischen Juristen in ihrem Billigkeitsgefühl nicht verstanden haben würden"; vgl. schon Behrends, Durchbruch (Fn. 5), 253 u. Fn. 79 und ergänzend Falk, Gelehrter (Fn. 3), 56-63.

12 Joachim Rückert, Der Geist des Rechts in Jherings «Geist» und Jherings «Zweck», in: Rg 5 (2004), 128149, 133 f.; der zweite Teil dieses Beitrags in Rg 6 (2005), 122-142.

13 Jhering, Sinn (Fn. 2), 461; 468 f. i. V. m. 481: "Um ihr (i. e. der unerträglichen Consequenz unseres Satzes) zu entgehen, gibt es keinen andern Weg, als den von mir eingeschlagenen, nämlich die Beschränkung hineinzutragen: wenn der Verkäufer durch den Untergang der Sache Schaden erlitten hat. Es handelt sich folglich um einen Fall der s. g. restrictiven Interpretation ...".

14 Wolfgang Fickentscher/Ulrich Himmelmann, Rudoph von Jherings Einfluß auf die Dogmatik und Methode des Privatrechts, in: G. Luf/W. Ogris (Hg.), Der Kampf ums Recht, Berlin 1995, 95-115, 96 f. i. V.m. $100 \mathrm{f}$.

15 Vgl. im Übrigen zur häufig angeführten Episode, dass Jhering später auch "in einem skizzierten Lebenslauf für die Reichstagswahlen 1867 in die Rubrik Religion einsetzte: «Romanist»" Behrends, Durchbruch (Fn. 5), $243 \mathrm{f}$.

16 Da der Erbschaftsverkauf durch die Vereinbarung sog. stipulationes emptae (et) venditae hereditatis ergänzt ist, findet sich dieser Text bei Paulus unter der Rubrik. De stipulationibus; vgl. Otto Lenel, Paligenesia Iuris Civilis, vol. I, Leipzig 1889, 1215 f.; zum Werk des Juristen etwa Justus Schmidt-Ott, 


\section{PENNITZ}

... sed hoc in re singulari non potest credi $^{17}$ : nam si eundem hominem tibi vendidero et necdum tradito eo alii quoque vendidero pretiumque accepero, mortuo eo videamus ne nihil tibi debeam ex empto ${ }^{18}$, quoniam ${ }^{19}$ moram in tradendo non feci ${ }^{20}$ (pretium enim hominis venditi non ex re, sed propter negotiationem percipitur $)^{20}$ et sic sit $^{21}$, quasi alii non vendidissem: $\langle\text { Lücke ? }\rangle^{22}$ tibi enim rem debebam, non

Pauli Quaestiones. Eigenart und Textgeschichte einer spätklassischen Juristenschrift, Berlin 1993, 1520.

17 Schmidt-Ott, Quaestiones (Fn. 16), 136 sieht diesen Einleitungssatz, der bei Jhering, Sinn (Fn. 2), 454 im Übrigen nicht abgedruckt ist, als interpoliert an, doch bezieht sich sed ... in re singulari unmittelbar auf das davor im Text stehende hereditatis, so dass eine nachklassische Textveränderung nicht plausibel erscheint; und ebensowenig das von Theodor Mommsen, Digesta Iustiniani Augusti, vol. I, Berlin 1866, 535 Fn. 2 in esset umgedeutete und zum davor stehenden Satz gezogene sed. Der Satz lautet: sed ubi hereditatem vendidi et postea rem ex ea vendidi, potest videri, ut negotium eius agam quam hereditatis. Eine Übersetzung von eius und hereditatis als, Genetiv der Beziehung' ("Sobald ich aber eine Erbschaft verkauft habe und später eine daraus stammende Sache verkauft habe, kann in Betracht kommen, dass ich das auf die Sache bezogene Geschäft wie ein auf die Erbschaft bezogenes führe") würde auch die von der neueren Lehre angeregten Textergänzungen zu diesem Satz überflüssig machen: Vgl. JeanFrançois Gerkens, «Aeque perituris». Une approche de la causalité dépassante en droit romain classique, Liège 1997, 220 f. und in diesem Sinn schon Martin Pennitz, Das periculum rei venditae. Ein Beitrag zum aktionenrechtlichen Denken im römischen Privatrecht, Wien u. a. 2000, 108 f. u. Fn. 19.

18 Bei Jhering, Sinn (Fn. 2), 454 steht hier emto. In der Rekonstruktion der Stelle bei Gerhard von Beseler, Romanistische Studien. Die Gefahrtragung beim Kaufe im klassischen Recht, in: TR 8 (1928), 279-313, 293 endet im Übrigen mit dem Wort empto der klassische Gehalt dieses Textteils, den er als "offenbar stark verbreitert" ansieht; vgl. sofort unten bei Fn. 22.

19 Berthold Kupisch, Tibi enim rem debebam, non actionem (Paulus D. 18, 4, 21). «Normzweck» im römischen Kaufrecht?, in: Mélanges C. A. Cannata, Bâle u. a. 1999, 37-51,40 u. Fn. 6 verweist auf die anderen Lesarten quando (Mommsen) und quotiens (nach der Mailänder Digestenausgabe), hält aber m. E. zu Recht an quoniam fest (ebd., 46 Fn. 19).

20 Jhering, Sinn (Fn. 2), 454 setzt nach feci einen Strichpunkt, verzichtet auf die Klammer und ergänzt nach percipitur einen Punkt; ähnlich Stefan Weyand, Kaufverständnis und Verkäuferhaftung, in: TR 51 (1983), 225-269, 259, der jedoch den Satz nach percipitur weitergehen lässt. Als nachklassischer Einschub wird der Klammerausdruck von Ingo Reichard, Die Frage des Drittschadensersatzes im klassischen römischen Recht, Köln u. a. 1993, 84 aufgefasst, der darin eine "didaktisch anmutende Kategoriebildung" sieht (dazu unten bei Fn. 67); zustimmend Martin Schermaier, Rez. Jakobs, in: ZRG RA 115 (1998), 555 u. Fn. 24. Dagegen zu Recht Hans G. Ullrich, Doppelverkauf und stellvertretendes commodum. Von D. 18.4.21 zu § 281 BGB (Diss. Bonn), Bonn 1990, 6 i. V. m. 51 f.; Horst H. Jakobs, lucrum ex negotiatione. Kondiktionsrechtliche Gewinnhaftung in geschichtlicher Sicht, Tübingen 1993, 10 i. V. m. 76 f. oder Kupisch, Normzweck (Fn. 19), 51 Fn. 33; ferner Gerkens, Approche (Fn. 17), 223 Fn. 44 f. (mit Lit.).

21 Jhering, Sinn (Fn. 2), 454 liest allerdings fit; anders schon Friedrich Mommsen, Erörterungen über die Regel: Commodum ejus esse debet, cujus periculum est, Braunschweig 1859, 110 Fn. 5; ebd., Fn. 6 findet sich dann auch die oft zitierte Kritik an Jherings Abhandlung von 1844 ("Es zeigt sich daran, wie viel Bestechendes die strenge Consequenz hat, selbst wenn sie zu den unrichtigsten Resultaten führt"), die schon dieser selbst andeutet (s. oben Fn. 11), doch reagierte Jhering, Sinn (Fn. 2), 456 f. u. Fn. 8 f. darauf dann doch eher verschnupft.

22 Zu Recht hebt Schmidt-Ott, Quaestiones (Fn. 16), 21 f. hervor, dass "Zitate anderer Juristen ... besonders häufig aber in den Quaestiones vorkommen", was "auch für die entsprechenden Werkgattungen bei Paulus" gilt. Es ist daher m. E. - wie noch näher auszuführen sein wird (s. unten Fn. 61 ff.) - nicht unwahrscheinlich, dass die Kompilatoren seine Auseinandersetzung mit anderen Juristenmeinungen vor dem Schlusssatz weggekürzt und dabei eventuell den Klammerausdruck (s. oben Fn. 20) aus dessen Kontext vorgezogen haben. S. auch die Ausführungen bei Jakobs, lucrum (Fn. 20), 73 f.: "Wir verstehen nur und 


\section{EIN "IRRTHUM UND DIE MÖGLICHKEIT, IHN SELBST ÖFFENTLICH ZURÜCKZUNEHMEN"}

actionem $^{23} \ldots$

... Aber das kann bei einer einzelnen Sache nicht angenommen werden ${ }^{24}$ : Wenn ich nämlich denselben Sklaven dir verkauft hatte und ihn, als er noch nicht übergeben war, auch einem Dritten verkaufte und den Preis in Empfang nahm, bleibt nach dessen Tod zu überlegen, $\mathrm{ob}^{25}$ ich dir (deshalb) nichts aus der actio empti schulde ${ }^{26}$, weil ich keinen Leistungsverzug begangen habe - denn den Preis für den verkauften Sklaven habe ich nicht aufgrund der Sache, sondern aufgrund eines Geschäftsabschlusses erhalten - und es (daher) so (anzusehen) sei, als hätte ich nicht an einen Dritten verkauft: <Lücke ?> Jedenfalls schuldete ich dir (damals) die Sache und keine Klage ${ }^{27} \ldots$

Anders als im Rechtsfall, den Jhering 1859 als Mitglied der Gießener Spruchfakultät zu entscheiden hatte ${ }^{28}$, geht Paulus also vom Doppelverkauf eines bestimmten Sklaven aus, wozu er bloß anführt, dass (1) die traditio an den ersten Käufer (K1) nicht erfolgte, dass (2) der Verkäufer (V) das pretium vom zweiten Käufer (K2) entgegennahm und dass (3) der

allerdings nicht, wie in der Entwicklung des Gedankens, um die es Paulus geht, ... all dies ohne jede Erläuterung gelassen und also vorausgesetzt werden kann. ... Wir finden gerade darin ... den Beweis, daß auch und gerade hier die Überlieferung uns nur die Spitze des Ganzen sehen läßt - nur das Resultat, das in schlechten Zeiten allein zählt und brauchbar scheint".

23 Zur Interpolationenkritik an diesem Satz vgl. insbes. Gerkens, Approche (Fn. 17), 223 f. u. Fn. 44-47, freilich mit dem gleichzeitigen Hinweis: "... le fond est généralment reconnu comme étant classique"; zur inhaltlichen "Unstimmigkeit", die zur Kritik am Schlusssatz geführt hat, ferner Reichard, Frage (Fn. 20), 85 f. u. Fn. 29 f.

24 S. zum Zusammenhang oben Fn. 17.

25 Ne lässt sich nach den Verben "(zu)sehen, erwägen, untersuchen" im Sinn von "ob etwa" verstehen; vgl. Karl E. Georges, Ausführliches lateinisch-deutsches Handwörterbuch, Bd. II, 8. Auf., Leipzig 1918, 1115 f. (II Anm. 2) bzw. Johann B. Hofmann/Anton Szantyr, Lateinische Syntax und Stilistik, München 1965, 542 (II a); s. etwa Iav. (5 ex post. Lab.) D. 41.2 .51 (arg. sed videamus, inquit, ne haec ipsa corporis traditio est, ...) od. Ulp. (23 ad Sab.) D. 34.3 .3 (arg. videamus, ne per acceptilationem debeam liberari: alioquin, ...); ferner Cic. Tusc. 3.13 (sed videamus ne hoc oratio sit hominum adsentantium ...). In diesem Sinn auch Okko Behrends u. a., Corpus Iuris Civilis. Text und Übersetzung III, Heidelberg 1999, 493; als Entscheidung des Paulus übersetzen diese Worte hingegen Schmidt-Ott, Quaestiones (Fn. 16), 136 Fn. 139; Kupisch, Normzweck (Fn. 19), 40 f. u. Fn. 7 bzw. Sylvia Sella-Geusen, Doppelverkauf. Zur Rechtsstellung des ersten Käufers im gelehrten Recht des Mittelalters, Berlin 1999, 37; in letzterem Sinn auch Ullrich, Doppelverkauf (Fn. 20), 8, der von einer "Frage ... doch wohl nur im rein grammatikalischen Sinn" spricht: "Nach dem Sinn des Gebrauchs von videamus ne liegt hier eine emphatisch gefaßte Feststellung vor" (ebd., 44 u. Fn. 91).

26 Die Verneinung non debere bezieht sich hier nicht nur auf die vertragliche Schuld, sondern auch auf ein allfälliges Interesse, wie das ja schon aus dem Hinweis auf die nicht vorliegende mora deutlich wird; vgl. in ähnlichem Sinn auch Ulp. (32 ad ed.) D. 19.1.11.18: Qui autem habere licere vendidit, videamus quid debeat praestare. ... pretium quidem deberi re evicta, utilitatem non deberi: neque enim bonae fidei contractus hac patitur conventione, ut emptor rem amitteret et pretium venditor retineret....

27 Ähnlich die Übersetzung des Schlusssatzes bei Kupisch, Normzweck (Fn. 19), 46 i. V. m. 41: Ich "schuldete dir ja eine Sache, nicht aber eine Klage".

28 Jhering, Sinn (Fn. 2), 452 f.; sein diesbezügliches Gutachten ist abgedruckt bei Kroppenberg, Plastik (Fn. 5), 60-88. Vgl. auch allg. Diethelm Klippel/Cordelia Kröger-Schrader, Rudof von Jhering an der Juristischen Fakultät der Ludwigs-Universität Gießen (1852-1868), in: O. Behrends (Hg.), Rudolf von Jhering. Beiträge und Zeugnisse, 2. Aufl., Göttingen 1993, 31-37, bes. 34 u. Fn. 58-60. 


\section{PENNITZ}

Sklave vor Eintritt eines Leistungsverzuges gegenüber K1 auf natürliche Weise starb. Angesichts dessen überlegt der Spätklassiker, ob K1 mittels actio empti nichts erlangen kann und es sich, da er das bei V befindliche pretium (des K2) nicht fordern könne, bezogen auf seine Rechtsposition so verhalte, als ob der zweite Verkauf gar nicht stattgefunden habe. Abschließend hält er fest, dass V die Ware schuldete, aber keine Klage, wobei hier das Imperfekt auffällt ${ }^{29}$.

Aus romanistischer Sicht erscheinen in Jherings Ausführungen zu D. 18.4.21 zwei Punkte besonders bemerkenswert: Zum einen muss er - im Hinblick auf die Abhandlung aus 1844 und auf seine bisherige Sicht der Frage - "einen Irrthum berichtigen, der, so lange er anhielt, den Weg zum Richtigen völlig versperrte": Denn im Text findet sich kein Hinweis darauf, dass "der Verkäufer von jedem der mehren Käufer den Kaufpreis fordern könne": "Paulus spricht in der ganzen 1.21 nur von der act. emti. Der act. venditi zu gedenken, hatte er weder eine Veranlassung, noch hat er es gethan"30. Diese Erkenntnis war bahnbrechend, Bernhard Windscheid etwa übernimmt sie sofort ${ }^{31}$, sie wird in der Jhering-Forschung betont ${ }^{32}$ und ist auch Ausgangspunkt der neueren römisch-rechtlichen Arbeiten zum Thema ${ }^{33}$ : Zugleich eröffnet sie mangels expliziter Quellenzeugnisse allen Interpreten den Spielraum ${ }^{34}$, im Fall eines Doppelverkaufs entweder von zwei zu gewährenden actiones venditi auszugehen ${ }^{35}$

29 Das betont zu Recht Reichard, Frage (Fn. 20), 85, wobei aber die Mitvergangenheitsform m. E. noch nicht "impliziert, daß die Obligation mit dem Untergang der Sache erloschen ist"; s. dann unten bei Fn. 69 ff.

30 Jhering, Sinn (Fn. 2), 453 f. Schon davor (ebd., 451) wird - wie es im Titel dieses Beitrags aufscheint "zu meiner Entschuldigung" angeführt, dass auch die bisherigen "Schriftsteller ... meinen Irrthum getheilt und mir damit zu meiner Freude die Möglichkeit offen gelassen haben, ihn selbst zuerst als solchen zu erkennen und öffentlich zurückzunehmen".

31 Bernhard Windscheid, Lehrbuch des Pandektenrechts, Bd. II.2, Düsseldorf 1866, 87 (§ 390) Fn. 17: "Vor allen Dingen ist nun Jhering darin beizu stimmen, daß der Verkäufer nicht von beiden Käufern das Kaufgeld fordern darf. Daß er es dürfe, sagt nicht die 1. 21 D. de her. vel act. vend. 18. 4 ..."; ebenso noch ders./Theodor Kipp, Lehrbuch des Pandektenrechts, Bd. II, 9. Aufl., Frankfurt/Main 1906, 666 (§ 390) Fn. 17.

32 Vgl. Behrends, Durchbruch (Fn. 5), 253 u. Fn. 78 oder Falk, Gelehrter (Fn. 3), 54 u. Fn. 248.

33 S. insbes. Ullrich, Doppelverkauf (Fn. 20), 58 u. Fn. 139 unter Berufung auf Jhering; ferner SchmidtOtt, Quaestiones (Fn. 16), 139 ("Der entscheidende Gesichtspunkt für diesen Sachverhalt ist die Haftung ex empto"); Schermaier, Rez. Jakobs (Fn. 20), 553; Kupisch, Normzweck (Fn. 19), 45 ("Des Paulus Überlegungen kreisen allein ... um eine Haftung aus Kauf"). In diesem Sinn wohl auch Sella-Geusen, Doppelverkauf (Fn. 25), 40 u. Fn. 175.

34 Die von Jakobs, lucrum (Fn. 20), 70 u. Fn. 135 diesbezüglich an Jhering geübte Kritik, man dürfe trotz der Konzentration der Entscheidung auf die actio empti "Paulus ... aus dieser Frage der Gefahrtragung bei zweimaligem Verkauf derselben Sache nicht überhaupt herauslassen", geht allerdings an der Sache vorbei: da Jhering seine Lösung ja gerade ausgehend von "Sinn" der Gefahrtragungsregel entwickelt, die dann natürlich auch bei Paulus zugrunde liegen würde; zudem nützt Jakobs das Schweigen des Spätklassikers bezüglich einer allfälligen actio venditi (gegen K1) in vergleichbarer Weise für den eigenen, von Jhering (dazu ebd., 71 Fn. 137 f.) freilich abweichenden Erklärungsansatz (dazu unten bei Fn. 54).

35 So etwa schon Behrends, Durchbruch (Fn. 5), 253 u. Fn. 78; ferner Ullrich, Doppelverkauf (Fn. 20), 6062; Gerkens, Approche (Fn. 17), 229 f.; Mario Talamanca, Considerazioni sul «periculum rei venditae», in: Seminarios Complutenses 7 (1995), 217-296, 285 f. Fn. 239 oder Pennitz, Periculum (Fn. 17), 110 f. 


\section{EIN "IRRTHUM UND DIE MÖGLICHKEIT, IHN SELBST ÖFFENTLICH ZURÜCKZUNEHMEN"}

oder aber bloß von einer ${ }^{36}$ oder gar von überhaupt keiner ${ }^{37}$.

Zum anderen ist es aus der Perspektive der heutigen Romanistik durchaus aufschlussreich, dass die meisten neueren Erklärungsansätze ${ }^{38}$ zum "Grund" des periculum emptoris-Satzes schon in Jherings Beitrag aufzufinden sind ${ }^{39}$ : So etwa die "aktionenrechtliche Sichtweise" der Frage ${ }^{40}$, die These einer Vorverlagerung der Wirkung des Eigentumserwerbs inter partes, die den Grundsatz res perit domino auf den Käufer erstrecken würde ${ }^{41}$, oder die Rückführung der Gefahrtragungsregel auf den altrömischen Barkaufgedanken, wofür sich dann Jhering selbst ausspricht ${ }^{42}$.

\section{Paulus als missverstandener römischer Jurist?}

Im Folgenden soll - angeregt durch Jherings scharfsichtige Analyse von D. 18.4.21 - eine von der bisherigen Lehre abweichende Interpretation dieses Textes vorgeschlagen werden. Denn ob man Paulus nun mit Jhering als "Puchta des römischen Alterthums" ansieht ${ }^{43}$, ihn

36 So Jhering, Sinn (Fn. 2), aufgrund des "Sinns" der periculum emptoris-Regel (dazu auch unten bei Fn. 53); ähnlich dann Windscheid, Lehrbuch (Fn. 31), 87 (§ 390/3) u. Fn. 17 (für Gefahrtragung des K1) bzw. ders./Kipp, Lehrbuch (Fn. 31), 666 (§ 390/3) u. Fn. 17 (für Gefahrtragung des K2).

37 So Jakobs, lucrum (Fn. 20), 71 f.; zustimmend Reichard, Frage (Fn. 20), 84 u. Fn. 28 sowie Schermaier, Rez. Jakobs (Fn. 20), 552.

38 Aus neuerer Sicht zu ergänzen wäre hier v. a. die These von Talamanca, Considerazioni (Fn. 34), passim, der bezüglich der Gefahrtragungsfrage bei der emptio venditio von ius controversum ausgeht.

39 Jhering, Sinn (Fn. 2), 462 f. u. Fn. 16 f.

40 So schon Carl G. v. Wächter, Ueber die Frage: Wer hat bei Obligationen die Gefahr zu tragen?, in: AcP 15 (1832), 97-138/188-222, 189 ff., bes. 194 f.; in diesem Sinn zuletzt Wolfgang Ernst, Die Einrede des nicht erfüllten Vertrags, Berlin 2000, 51 f.; 56 f. Etwas anders Pennitz, Periculum (Fn. 17), 74-82, 75 f.; 95 f. was Jhering, Sinn (Fn. 2), 462 hoffentlich von der dort geäußerten Kritik abhalten würde, dass der eingangs genannte Ansatz eben "zu der Art und Weise, wie die römischen Juristen sonst das Verhältnis zwischen Leistung und Gegenleistung bei den zweiseitigen Contracten auffassen, nicht stimmt".

41 So schon Windscheid, Lehrbuch (Fn. 31), 87 (§ 390) Fn. 17; ders./Kipp, Lehrbuch (Fn. 31 ), 330 f. (§ 321) u. Fn. 19a; vgl. ferner etwa Ullrich, Doppelverkauf (Fn. 20), 50 u. Fn. 112 f.; Schmidt-Ott, Quaestiones (Fn. 16), 139 u. Fn. 152; Jakobs, lucrum (Fn. 20), 71; Martin Bauer, Periculum emptoris, Berlin 1998, 75-79 (mit Lit.) und wohl auch Kroppenberg, Plastik (Fn. 5), 26 Fn. 71; 32.

42 Jhering, Sinn (Fn. 2), 463 f.; übernommen findet sich dieser Gedanke bei Wolfgang Kunkel/Heinrich Honsell, Römisches Recht, 4. Aufl., Berlin u. a. 1987, 310. Zu einer diesbezüglichen Verschmelzung der Ansätze von Jhering und Windscheid kommt es bei Max Kaser, Das römische Privatrecht, 2. Aufl., Bd. I, München 1971, 552 u. Fn. 63, hingegen zu einer anderen Art einer solchen Verschmelzung bei Fritz Schulz, Classical Roman Law, Oxford 1951, 553, wonach der Verkäufer "derserved a recompense" (s. unten Fn. xy).

43 Vgl. Rudolf v. Jhering, Der Besitzwille. Zugleich eine Kritik an der herrschenden juristischen Methode, Jena 1889, 282 f.: "Von diesem souveränen Übermuth des sich in sich selbst zurückziehenden juristischen Denkens gegenüber dem positiven Recht ... giebt derselbe Jurist noch bei einer anderen Frage einen Beleg: ... ,Was ich nicht construiren kann, / Seh ich als nicht vorhanden an!' ... ist geeignet die Charakteristik des Manns zu vervollständigen. ... Ich glaube im Bisherigen den Leser zur Genüge in Stand gesetzt zu haben, sich ein Urtheil über die wissenschaftliche Individualität des Paulus zu bilden. Mich erinnert derselbe am meisten an Puchta - es ist der Puchta des römischen Alterthums". In dieser 


\section{PENNITZ}

- wie Kupisch das tut - als dunklen Juristen bezeichnet ${ }^{44}$ oder solche Zuschreibungen im Einklang mit Jakobs entschieden in Abrede stellt ${ }^{45}$, die ganz unterschiedlichen Erklärungsansätze der bisherigen Lehre ${ }^{46}$ zu diesem Teilstück von D. 18.4.21 machen doch zur Genüge deutlich, dass sich der Gedankengang des Spätklassikers mangels erläuternder Ausführungen seinerseits ${ }^{47}$ jedenfalls missverstehen lässt. Insofern soll hier - freilich in vielen Teilaspekten gestützt auf die beeindruckenden Auslegungen von $\mathrm{Jakobs}^{48}$ und $\mathrm{Kupisch}^{49}$, die wiederum auf die qualitätsvollen Überlegungen von Ullrich und Reichard bzw. SchmidtOtt und Gerkens aufbauen können ${ }^{50}$, die Frage aus aktionenrechtlicher Sicht betrachtet werden: Somit wird sich der Lösungsansatz - anders als bei Jhering oder Jakobs, die letztlich bei den actiones venditi des Doppelverkäufers ansetzen - auf die von Paulus selbst ins Zentrum gerückte actio empti konzentrieren (arg. videamus, ne nihil debeat ex empto).

leidenschaftlichen Kritik an Paulus wirkt wohl, wie der Hinweis (ebd., 283 Fn. 1) auf "Scherz und Ernst" (dazu schon bei Fn. 7) belegen dürfte, auch das Missvergnügen des Gelehrten nach, durch diesen Juristen und dessen "Consequenz" zu besagtem "Irrthum" verführt worden zu sein; als weiteren Anhalt für diese These s. ebd., 300: "Paulus ... hat sich auch bei dieser Gelegenheit wiederum als Denjenigen dargethan, als welchen wir ihn bereits früher haben kennen lernen: als Doctrinär vom reinsten Wasser, der seine fixen Ideen durchführt, es koste, was es wolle. Und diesem Manne ist die moderne Jurisprudenz gläubig und kritiklos gefolgt, ohne gleich ihm die mindeste Notiz von dem positiven Recht zu nehmen - die ächte Jüngerin der von ihm inaugurirten ungesunden Begriffsjurisprudenz".

44 Kupisch, Normzweck (Fn. 19), 49 i. V. m. 38 f., unter Bezugnahme auf dens., Id quod interest bei Nichterfüllung und Verzug des Verkäufers, in: TR 43 (1975), 1-22, bes. 14-16 sowie dens., D. 46,2,12 (Paulus 31 ad edictum). Zum Subjektswechsel als Auslegungsproblem, in: FS R. Lukes, Köln u. a. 1989, 743754, 754, jeweils mit weiteren Hinweisen.

45 Jakobs, lucrum (Fn. 20), bes. 50 f. i. V. m. 44 f. u. Fn. 76 sowie insbes. ebd., 69 (dazu unten Fn. 52) bzw. 80: "Wir müssen nur die Fälle richtig haben, und wir wissen immer, was richtig ist. Dies lehrt uns Paulus, und dies ist die Lehre des Rechts der römischen Juristen, die uns eben darum Klassiker sind und es nicht mehr sein können in einer Zeit, die wenn's um das Recht geht, gar nicht mehr weiß, was richtig ist: die in allem Recht ein Moment inhaltlicher Willkür findet".

46 So hält Sella-Geusen, Doppelverkauf (Fn. 25), 45 bezüglich der Gefahrtragungsfrage zu Recht fest: "Bis heute ist unsicher, wie sie nach römischem Recht gelöst wurde"; in diesem Sinn ist wohl auch Wolfgang Ernst, Doppelverkauf. Ein Panorama, in: FS A. Heldrich, München 2005, 113-142, 118 f. sowie ders., Der zweifache Verkauf derselben Sache - Betrachtungen zu einem Rechtsproblem in seiner europäischen Überlieferung, in: E. Jakab/ders. (Hg.), Kaufen nach Römischem Recht, Berlin-Heidelberg 2008, 83-103, $100 \mathrm{f} . \mathrm{zu}$ verstehen.

47 S. schon oben Fn. 22.

48 Das gilt vor allem für den von Jakobs, lucrum (Fn. 20), 72 f. skizzierten, alternativen Erklärungsansatz ("Wem aber dies alles eine zu formale Argumentation ist, der kann sich die Sache auch so zurechtlegen: ..."), doch wird das Grundproblem weiterhin bei der Gefahrtagungsregel, d. h. bei der actio venditi verortet: "Übergabe und Zahlung plus Untergang der Sache sind die beiden die Gefahrtragung beim Doppelverkauf entscheidenden Akte".

49 Das betrifft etwa das Verständnis von actio bei Kupisch, Normzweck (Fn. 19), 48 (s. auch unten Fn. 60) im Schlussatz von D. 18.4.21 (tibi enim rem debebam, non actionem), womit wohl zugleich - ohne das freilich "öffentlich" zu machen (ebd., 44 f.) - gegen die Interpretation von Okko Behrends in: Corpus Iuris Civilis III (Fn. 25), 493 Fn. 1; 494 Fn. 2 (s. auch ders., Scritti «italiani», Napoli, 2009, 346 Fn. 209) Stellung bezogen wird, der - ähnlich wie Weyand, Kaufverständnis (Fn. 20), 263 f. - actio hier mit "Handlung" übersetzt.

$50 \quad$ S. schon oben Fn. 16 f. u. 20. 


\section{EIN "IRRTHUM UND DIE MÖGLICHKEIT, IHN SELBST ÖFFENTLICH ZURÜCKZUNEHMEN"}

Aber auch ein solche Erklärung wird sich gegen den Vorwurf, die Worte von Paulus misszuverstehen, nicht absichern lassen, da man für ein Verständnis von D. 18.4.21 manches vorauszusetzen hat, was den Quellen so nicht zu entnehmen ist ${ }^{51}$, und sich zudem wohl kaum der wertenden Herangehensweise entziehen $\mathrm{kann}^{52}$ : Und ähnlich wie Jhering die Preisgefahr des Käufers (im Regelfall) als Ersatz bzw. Schadloshalten im Sinn einer "Versicherungssumme" zu Gunsten des verkaufenden Eigentümers beim zeitlich gestreckten Geschäft konstruiert, ${ }^{53}$ bzw. Jakobs den Doppelverkauf prinzipiell mit einem Rückfall jeglicher Gefahrtragung auf den Verkäufer verknüpft, weil der emptor nach römischem Recht inter partes bereits als Eigentümer anzusehen sei $^{54}$, um jeweils zu einer (subjektiv) als billig eingestuften

51 Vgl. in diesem Sinn auch Jhering, Sinn (Fn. 2), 480: "Wer unter Begründung ausdrückliche Quellenäußerungen versteht, wer nur Stellen, nicht aber die Deduction aus der innern Consequenz eines Rechtsinstituts als Beweismittel anerkennt, den zu gewinnen werden wir freilich von vornherein verzichten müssen. Denn Stellen gibt es hier nicht; sonst wäre die hier aufgestellte Theorie wohl schon längst vorgetragen worden"; man habe daher bei der Lösung darauf zu achten, "dass uns die Quellen wenigstens nicht entgegenstehen".

52 Jhering, Sinn (Fn. 2), 461 verweist insofern darauf, dass "sich das gesunde Rechtsgefühl gegen die doppelte Zahlung des Kaufpreises entschieden auflehnt", während Jakobs, lucrum (Fn. 20), 69 - nicht weniger eindringlich - bekennt: "Da Paulus unser guter Geist sein muß, da wir die Wahrheit bei ihm als vorhanden voraussetzen, kann er - vor Zahlung des Kaufpreises - dem Verkäufer nicht die Kaufpreiszahlungsklage gegen beide Käufer zugestanden, also nicht beide mit der Gefahr belastet haben".

53 Denn wie Jhering, Sinn (Fn. 2), 465 anführt, sei ja der Verkäufer nach Vertragsabschluss "der Möglichkeit beraubt, durch anderweitige Disposition über die Sache den Zufall von sich abzuwenden (s. ferner ebd., 486); dazu auch Behrends, Durchbruch (Fn. 5), 254. Falk, Gelehrter (Fn. 3), 70 f. sowie Seinecke, Methode (Fn. 5), 173 (Rn. 422) interpretieren diesen Ansatz dahingehend, dass "Jhering ... aus der Regel über die Gefahrtragung für die Gegenleistung eine Schadensersatznorm" mache, doch sollte man dann besser auf ein "Zurechnungskriterium der Sphäre" hinweisen (vgl. nur den von Jhering herangezogenen Beleg D. 19.2.19.9, 479), da es ja am kausalen Zusammenhang von (typischerweise vorliegender) culpa (des Käufers am Traditionsaufschub) und casus-Eintritt fehlt. Jedenfalls folgert Jhering (ebd., 467 f.; 475 f.) für den Ausnahmefall des Doppelverkauf, dass ein solcher Ersatz ebenso wie eine Versicherungssumme nur einmal zustehen dürfe, weshalb eine zweimalige Forderung des jeweils vereinbarten pretium ausgeschlossen sei: Vielmehr komme dem Verkäufer ein Wahlrecht zu, welchen der beiden Käufer er belangen will; allerdings räumt Jhering (ebd. 478) selbst ein, dass "dies Wahlrecht etwas Mißliches hat". Behrends, Rechtsgefühl (Fn. 5), 74 u. Fn. 20 nennt es etwa einen "Schönheitsfehler der Jheringschen Lösung" und Jakobs, lucrum (Fn. 20), 63 Fn. 115 erinnert in diesem Zusammenhang an die berüchtigte "Inversionsmethode".

54 Jakobs, lucrum (Fn. 20), 70 f.: "... wenn also alles, was den neueren Interpreten als Lösung dazu eingefallen ist, für Paulus nicht in Betracht kommt, dann bleibt für ihn nur die Ansicht übrig, daß bei mehrfachem Verkauf derselben Sache der Verkäufer auf Grund des bloßen Vertragsschlusses den Kaufpreis von keinem der mehreren Käufer verlangen und doch den zuerst gezahlten Preis behalten kann. ... Wie immer das periculum emptoris zu erklären sein mag, es liegt in ihm jedenfalls eine Vorverlagerung der Wirkung des Eigentumserwerbs: es gilt res perit domino für den Käufer, schon bevor er Eigentümer der Kaufsache ist. Also kann ebensowenig wie zwei Käufer derselben Sache jeder für sich an dieser das Eigentum erwerben können, die in diesem Gefahrübergang vorgezogene Wirkung des Eigentumserwerbs bei mehreren Käufern für jeden gesondert eintreten": Merkwürdig ist dabei allerdings, dass V das von K2 entgegen genommene pretium "behalten kann", Jakobs (ebd., 72) muss hier von einem Gefahrtragungs(rück)übergang auf jenen Käufer ausgehen, "der so dumm war für etwas zu zahlen, was er nicht in die Hand bekommen": Trotz einhelliger Zustimmung von Reichard, Frage (Fn. 20), 84 f. Fn. 28 und Schermaier, Rez. Jakobs (Fn. 20), 552 scheint mir ein solcher Rekurs auf 


\section{PENNITZ}

Lösung zu gelangen, gründet sich auch die hier vorgelegte Auslegung auf letztlich nicht "beweisbare" Annahmen.

Wie schon Kupisch und ebenso Jakobs bezüglich der hier interessierenden Entscheidung zu Recht betonen, "kreisen des Paulus Überlegungen allein ... um eine Haftung aus Kauf"55. Doch darauf folgt m. E. bereits das erste Missverständnis: Denn es ist nicht so klar, wie es die bisherige Lehre annimmt, dass Paulus die Haftung an dieser Stelle verneint, ${ }^{56}$ der Spätklassiker wirft "im Kreise seiner Disputanten" ${ }^{57}$ bloß die Frage auf, ob V nach einem solchen Doppelverkauf tatsächlich nicht ex empto haftet ${ }^{58}$, und diese Frage zu stellen, heißt nach dem hier vertretenen Ansatz: sie zu verneinen ${ }^{59}$. Paulus bezweifelt also ganz konkret, ob V nach Erhalt des Kaufpreises von Seiten des K2 nun dem K1 nichts schulde, weil das pretium, das von V nicht im Zuge der Sachverfolgung ${ }^{60}$, sondern aus einem gesonderten Geschäft erlangt wurde, von K1 nicht an Stelle des toten Sklaven gefordert werden könne, und ob es deshalb so zu sehen sei, d. h. ob sich aus solcher Perspektive die unausweichliche Schlussfolgerung ergebe, dass die zweite emptio venditio des $\mathrm{V}$ hier rechtlich irrelevant sei.

In der komplexen, wohl durch einen (gedanklichen) Einschub ${ }^{61}$ ergänzten Frage spiegelt sich insofern - das ließe sich dann als zweites Missverständnis bezeichnen - nicht die Auffassung des Spätklassikers unter Einschluss allfälliger Begründungen seiner Ansicht wider ${ }^{62}$, er bezieht sich darin vielmehr auf eine etablierte Juristenmeinung, der man im Sinne

"Dummheit" (auch ebd., 77) bei Vertragstreue eines Käufers gleichfalls "etwas Mißliches" an sich zu haben (s. die vorige Fn.).

55 S. Kupisch, Normzweck (Fn. 19), 45 u. Fn. 16; so schon Jhering, Abhandlungen (Fn. 11), 59: "Paulus sagt ja nicht, daß der durch eine negotiatio vermittelte Gewinn keine Bereicherung sei, sondern, daß er nur da nicht herausgegeben zu werden brauche, wo Jemand für die Sache selbst hafte". Vgl. ferner Jakobs, lucrum (Fn. 20), 12 f.: "Das Fragment behandelt dann auch in seiner ganzen Länge ... eben die Haftung des Verkäufers: beim Verkauf einer Erbschaft zunächst, beim Verkauf sodann einer res singularis, ..."; der damit einhergehenden Kritik an Deutungen, die sich etwa nur auf den Doppelverkauf der res singularis konzentrieren und dadurch zu völlig falschen Schlussfolgerungen gelangen (ebd., $6 \mathrm{f}$. i. V. m. 13 Fn. 27), hoffe ich insoweit zu entgehen, als ich mich bereits an anderer Stelle - Pennitz, Periculum (Fn. 17), 103-117 - dem Gesamtzusammenhang widmen konnte und der hier vorgelegten Interpretation nur ergänzender Charakter zukommt (s. auch unten Fn. 83).

56 Vgl. Kupisch, Normzweck (Fn. 19), 45 zur "Entscheidung des Paulus, unter den gegebenen Voraussetzungen schulde der Verkäufer dem Erstkäufer ex empto nichts"; sowie ebd., 49 f.; s. ferner etwa Weyand, Kaufverständnis (Fn. 20), 261; 263 oder Jakobs, lucrum (Fn. 20), 57 f. i. V. m. 43 u. 56 f.; sowie ebd., 60 mit der m. E. korrekten Folgerung, dass Paulus hier keine traditio an K2 voraussetzt; dafür spricht wohl auch der bereits bei Pennitz, Periculum (Fn. 17), 110 Fn. 25 angeführte Beleg Proc. (6 epist.) D. 18.1.68.2.

57 So Jakobs, lucrum (Fn. 20), 12 und, vergnüglich zu lesen, die Rekonstruktion möglicher Debatten (ebd., 31 f.; 43 f.).

$58 \quad$ S. dazu schon oben bei Fn. 26.

59 Völlig konträr die bereits zitierten Ausführungen bei Ullrich (s. oben Fn. 25).

60 Vgl. auch Kupisch, Normzweck (Fn. 19), 49 i. V. m. 50 f. u. Fn. 33, der deshalb davon ausgeht, dass "Paulus dem aktuellen Fall des Singularverkaufs einer Sache hypothetisch den Fall der Singularverpflichtung eines Verkäufers auf Abtretung einer Klage auf die Sache gegenüber" stellt.

61 Zur Interpunktion in D. 18.4.21, die bereits eine Interpretation des überlieferten Textes - vgl. Justiniani augusti pandectarum codex florentinus, cur. A. Corbino/B. Santalucia, vol. I, Firenze 1988, 266r darstellt, bereits oben Fn. 20.

62 So etwa Jakobs, lucrum (Fn. 20), 43 f. i. V. m. 76-79. 


\section{EIN "IRRTHUM UND DIE MÖGLICHKEIT, IHN SELBST ÖFFENTLICH ZURÜCKZUNEHMEN"}

Jherings "strenge Consequenz" zuschreiben kann ${ }^{63}$ : Im Rahmen seiner quaestio dürfte Paulus wohl explizit auf andere Juristen verwiesen ${ }^{64}$ und zu deren Argumenten, zu denen auch der (vielleicht vorgezogene) Klammerausdruck gehört, Stellung bezogen haben, doch wurde dieser Teil eines langen Exkurses (zum zentralen Erbschaftsverkaufsfall) von den Kompilatoren gekürzt und auf die in diesem Kontext letztlich allein relevante Entscheidung des Paulus reduziert, nämlich auf den Satz: tibi enim rem debebam, non actionem ${ }^{65}$.

Weil kein Schuldnerverzug vorlag, hätte die von Paulus wiedergegebene, sozusagen "althergebrachte" Auffassung dem V - gestützt auf das periculum emptoris ${ }^{66}-$ in der Tat beide pretia zugesprochen. Allerdings käme gemäß einer solchen Sichtweise - mangels eines Verschuldens des V - eine Klagenabtretung durchaus in Betracht, diese wäre etwa im Fall eines zwischenzeitlich (von vierter Seite) verübten furtum zu bejahen ${ }^{67}$ : Deshalb hat man auch das von K2 erhaltene pretium näher zu charakterisieren, dieses sei aber deshalb nicht zu leisten, weil es sich aus einem vom Erstkauf unabhängigen zweiten Geschäftsabschluss des $\mathrm{V}$ herleite (arg. pretium enim ... propter negotiationem percipitur). Aus spätklassischer Sicht wird jene in D. 18.4.21 (eben bloß) zitierte Auffassung freilich nicht so ohne weiteres geteilt, wie ein vergleichbarer Fall verdeutlicht, bei dem Ulpian (arg. et verius est) ebenso wie Paulus die Vertragsklage auf ein pretium (ex negotiatione) mit der Hinweis gewähren, dass dessen Nichtherausgabe als schuldhaftes Verhalten anzusehen sei ${ }^{68}$.

Im vorliegenden Fall bedarf es jedoch keiner Anknüpfung an diesen (spätklassischen) Lösungsansatz, da Paulus m. E. von einer Vertragsverletzung des V gegenüber K1 ausgeht:

63 S. Jhering, Sinn (Fn. 2), 456 Fn. 8: "Ich hatte jene Consequenz nicht gezogen, sondern glaubte sie ... bei Paulus wahrzunehmen".

64 S. schon oben Fn. 22.

65 Gemäß Jakobs, lucrum (Fn. 20), 44 i. V. m. 79 f. wäre das hingegen der "3. Grund", wobei Paulus (wie beim Erbschaftsverkauf) von einer "actio gegen den Vierten" ausgegangen sei.

66 Dazu Pennitz, Periculum (Fn. 17), 69 ff., 94-98 sowie zum neueren Meinungsstand ebd., 36-47.

67 Vgl. Ulp. (29 ad Sab.) D. 47.2.14 pr.: Eum qui emit, si non tradita est ei res, furti actionem non habere, sed adhuc venditoris esse hanc actionem Celsus scripsit. mandare eum plane oportebit emptori furti actionem et condictionem et vindicationem, et si quid ex his actionibus fuerit consecutus, id praestare eum emptori oportebit: quae sententia vera est, et ita et Iulianus. et sane periculum rei ad emptorem pertinet, dummodo custodiam venditor ante traditionem praestet. Die vom schuldlosen Verkäufer (arg. dummodo custodiam venditor ante traditionem praestet) aufgrund der rei vindicatio erzielte litis aestimatio lässt sich durchaus als Erwerb ex re und zugleich als pretium hominis venditi auffassen, ohne dass man deshalb hierauf bezogen an eine "didaktisch anmutende Kategoriebildung" (s. oben Fn. 20) denken muss; vgl. nur Paul. (10 ad Sab.) D. 6.1.46: Eius rei, quae per in rem actionem petita tanti aestimata est, quanti in litem actor iuraverit, dominium statim ad possessorem pertinet: transegisse enim cum eo et decidisse videor eo pretio, quod ipse constituit. Das erklärt letztlich auch, warum in dem von Paulus davor in D. 18.4.21 angeführten Stipulationsbeispiel jeglicher Hinweis auf ein pretium accipere fehlt; diese Frage stellt sich beim iudicium stricti iuris nicht: ... non enim deberet Stichi promissor, si eum vendidisset, mortuo eo, si nulla mora processisset. ...; zur "Frage" auch Jakobs, lucrum (Fn. 20), 55 f.

68 Ulp. (30 ad ed.) D. 16.3.1.47 / Paul. (31 ad ed.) D. 16.3.2: Quia autem dolus dumtaxat in hanc actionem venit, quaesitum est, si heres rem apud testatorem depositam vel commodatam distraxit ignarus depositam vel commodatam, an teneatur. et quia dolo non fecit, non tenebitur de re: an tamen vel de pretio teneatur, quod ad eum pervenit? et verius est teneri eum: hoc enim ipso dolo facit, quod id quod ad se pervenit non reddit. I quid ergo, si pretium nondum exegit aut minoris quam debuit vendidit? actiones suas tantummodo praestabit. 


\section{PENNITZ}

Insofern verweist er in seiner Entscheidung, d. h. im letzten Satz des Exkurses darauf ${ }^{69}$, dass V schon zuvor nur die Sache schuldete, auf die sich dann letztendlich auch der Schadensersatzanspruch von K1 konkret bezieht: Das auffällige Imperfekt ${ }^{70}$ bezieht sich dabei genau auf jenes Faktum, das im Rahmen des überlieferten Textes unmittelbar vor dem Schlusssatz genannt ist, nämlich auf den Zweitverkauf, der aus Sicht von Paulus also sehr wohl zu berücksichtigen ist: Trotz dieser alii venditio schuldete $\mathrm{V}$ weiterhin ausschließlich die Sache, nicht etwa eine Klage: Eine solche könnte sich jedenfalls solange (arg. debebam) auf den mit K2 vereinbarten Kaufpreis richten, bis K2 die Vor-Leistung erbringt ${ }^{71}$, es könnte sich aber auch um eine Klage "auf die Sache" handeln, wenn etwa V den Sklaven in der Folge formlos tradiert ${ }^{72}$ (arg. tibi enim rem debebam, non actionem).

Damit stellt sich abschließend die zentrale Frage, worin Paulus die Vertragsverletzung des V gegenüber K1 erblickt haben könnte: Denn dass die bloße Tatsache eines Zweitverkaufs dafür keine hinreichende Grundlage bietet, hat schon Jhering völlig zu Recht festgehalten ${ }^{73}$ : Der Verkäufer könnte ja "jedem der beiden Käufer, der sich darauf beriefe, ... entgegnen, daß er gerade ihm geleistet haben würde"74. Der entscheidende Punkt liegt deshalb m. E. darin, dass V nach Abschluss der beiden Verträge von K2 das pretium entgegennimmt, wie es Paulus ja auch explizit festhält (arg. pretiumque accepero) ${ }^{75}$. In diesem auch nach außen hin wirksamen Akt kommt nämlich eine Präferenz bzw. electio des V zu Gunsten einer der

69 S. auch oben bei Fn. 65.

70 Vgl. schon oben bei Fn. 29.

71 Ob das der Fall sein kann, hängt freilich davon ab, ob man bei der Wendung alii quoque vendidero pretiumque accepero von einem Pränumerationskauf ausgeht, bei dem V den Preis sofort mit Vertragsabschluss erhält oder ob das pretium accipere erst etwas später erfolgt ist; beides ist m. E. vorstellbar.

72 Natürlich wäre eine solche Klagenzession "sinnlos", da K2 (als bonitarischer Eigentümer des Sklaven) die abgetretene rei vindicatio nun - im Sinn von Iul.-Ulp. (74 ad ed.) D. 44.4.4.32 - mittels exceptio (doli) entschärfen könnte, doch spricht dieser Umstand ja nicht gegen, sondern für das bei Paulus angeführte non actionem (debere). S. ferner zur Auslegung von Kupisch bereits oben Fn. 60; aber auch der von Jakobs in seine Überlegung einbezogene "Vierte", bei dem sich der Sklave befindet (s. oben Fn. 65), käme dabei allenfalls in Betracht.

73 Freilich hat man dabei vorauszusetzen, dass V nicht von vornherein nachweisbar dolos agiert, da sich die hier aufgeworfene Frage dann gar nicht stellt, vgl Jhering, Sinn (Fn. 2), 458-461; Jakobs, lucrum (Fn. 20), 72 oder Pennitz, Periculum (Fn. 17), 112 f. Fn. 34.

74 Jhering, Sinn (Fn. 2), 455 f.: "Ebenso hat der Verkäufer ganz nach allgemeinen Grundsätzen gegen beide [scil. Käufer] die act. venditi, d. h. sie können ihm nicht den doppelten Verkauf als solchen, sondern nur, wie überall, die exc. non adimpleti contractus entgegensetzen, insofern dieselbe begründet ist. ... Ist aber der Umstand, daß der Verkäufer doppelt verkauft hat, an sich rechtlich einflußlos, bestehen beide Contracte, so lange die Sache existirt,völlig unabhängig neben einander, ohne sich gegenseitig zu beeinträchtigen, warum sollte sich dies ändern, wenn die Sache untergeht? Freilich hätte der Verkäufer beiden Käufern nicht leisten können, allein die Frage von der Möglichkeit der doppelten Beschaffung der Leistung ist ja jetzt völlig erledigt; jedem der beiden Käufer, der sich darauf beriefe, könnte er entgegnen, daß er gerade ihm geleistet haben würde".

75 Zu Recht betont Jakobs, lucrum (Fn. 20), 67 u. Fn. 124, man müsse "für diese Vorleistung des 2. Käufers eine Erklärung geben". Allerdings muss es m. E. präziser heißen: für die Tatsache, dass Paulus an dieser Stelle auf den Zahlungsempfang des V abstellt (arg. vendidero pretiumque accepero). 


\section{EIN "IRRTHUM UND DIE MÖGLICHKEIT, IHN SELBST ÖFFENTLICH ZURÜCKZUNEHMEN"}

beiden von ihm abgeschlossenen emptiones venditiones zum Ausdruck ${ }^{76}$. Besonders deutlich zeigt sich das anhand einer aktionenrechtlichen Betrachtung: Indem V das pretium des K2 akzeptiert, räumt er letzterem eine "voraussetzungslose" Prozessführung mittels actio empti ein; anders verhält es sich hingegen bei K1, dem vom iudex weiterhin - im Sinn des grundsätzlichen Austauschcharakters der emptio venditio, auf den die demonstrationes der Klagen verweisen - das pretium offerre aufzuerlegen ist, um eine Verurteilung des $\mathrm{V}$ zu erreichen ${ }^{77}$. K1, dem ungeachtet des Vertragsschlusses zwischen V und K2 weiterhin der Sklave geschuldet wurde (arg. tibi enim rem debebam, non actionem), kann sich insofern ab dem Moment, ab dem $\mathrm{V}$ das pretium des K2 entgegengenommen hat, bezüglich dieses rem debere auf eine Vertragsverletzung des V berufen, da er einem anderen, d. h. dem K2, eine jederzeit durchsetzbare Klage auf die res vendita eingeräumt hat ${ }^{78}$. Die so beschriebene Rechtslage bliebe dann nach Auffassung des Spätklassikers, wenn sie denn "auch die des Paulus ist"79, bis zum Tod des Sklaven aufrecht und wird durch den eintretenden casus gleichsam irreversibel ${ }^{80}$, da $\mathrm{V}$ das empfangene pretium nun nicht länger - nämlich mit rechtlicher Wirkung bezüglich des Erstverkaufs - wieder an K2 rückübertragen kann: Denn mit Tod des Sklaven trifft K2 das periculum rei venditae ${ }^{81}$, während V weiterhin dem Schadensersatzanspruch des K1 ausgesetzt bleibt.

76 Einer traditio an K2 käme natürlich dieselbe Bedeutung zu, weshalb sie für den Exkurs von Paulus keine unmittelbare Relevanz hat und daher auch nicht erwähnt werden muss; s. ferner Jakobs, lucrum (Fn. 20), 73 i. V. m. 57 ff. Ähnlichkeiten mit einer solchen traditio weist m. E. die in Iul. (13 dig.) D. 19.1.23 diskutierte Freilassung eines (bloß) verkauften Sklaven zu sehen; auf diesen Text verweist im Übrigen schon Jhering, Abhandlungen (Fn. 11), 59 f. u. Fn. 1.

77 Vgl. zu Ulp. (32 ad ed.) D. 19.1.13.8 insofern Pennitz, Periculum (Fn. 17), 75-77.

78 Anders Jakobs, lucrum (Fn. 20), 73, der sich nur auf die (nicht erfolgte) traditio konzentriert. Sollte übrigens V den Sklaven um einen höheren Preis an K2 verkauft haben, was natürlich ein nachvollziehbares Motiv für den Zweitverkauf darstellen würde, so wäre dann dieses entgegengenommene pretium eine wichtige, aber andererseits nicht die einzige denkbare Bezugsgröße für die Höhe der Schadensersatzforderung.

79 Es darf hier auf Kupisch, Normzweck (Fn. 19), 51 verwiesen werden: "Die Meinung C [d. h. die von Kupisch] hat von der Rede des Paulus eine präzise inhaltliche Vorstellung. Freilich, ob sie auch die des Paulus ist? Nullibi rationes iuris magis profundae, nullibi peior interpretum causa, praecipue equidem, si cum Paulo res sit".

80 Eine vergleichbar irreversible Wirkung des Todes eines Sklaven zeigt sich etwa auch in Paul. (33 ad ed.) D. 18.1.34.6: Si emptio ita facta fuerit: , est mihi emptus Stichus aut Pamphilus', in potestate est venditoris, quem velit dare ..., sed uno mortuo qui superest dandus est: et ideo prioris periculum ad venditorem, posterioris ad emptorem respicit....

81 Insofern geht etwa schon Ernst Rabel, Gefahrtragung beim Kauf, in: RZG RA 42 (1921), 543-564, bes. 548 u. Fn. 1 m.E. völlig zu Recht davon aus, dass sich das periculum emptoris gerade anhand des Verhältnisses zwischen V und K1 zeigt; anders freilich Jhering, Sinn (Fn. 2), 486, der davon ausgeht, dass "durch den Casus ... die Erfüllung des Contracts von beiden Seiten unmöglich wird", und zwar im Einklang mit seiner zentralen These: "der Käufer versichert den Verkäufer, der Kaufpreis ist eine Versicherungssumme"; gegen beide Ansätze wiederum Jakobs, lucrum (Fn. 20), 70 f. u. Fn. 135 f. 


\section{PENNITZ}

\section{4. "Gesundes Rechtsgefühl" und Einzelfallbetrachtung}

Unter Berufung auf das "gesunde Rechtsgefühl" sowie den "gesunden Sinn der römischen Juristen"82 entwickelte also Jhering - gestützt auf den "Sinn" des periculum emptoris-Satzes, der freilich so den Quellen nicht zu entnehmen ist - eine grundlegende Lösung für das Gefahrtragungsproblem beim Doppelkauf. Die hier vorgelegte Interpretation eines darauf bezogenen Textabschnitts in Paul. D. 18.4.21 kann freilich einer solchen Zielsetzung nicht gerecht werden: Sie muss sich damit begnügen, dass Paulus anhand einer konkreten Fallkonstellation den "an sich" auch gegen K1 bestehenden Kaufpreisanpruch des (nicht dolosen) V dadurch konterkariert, dass sich K1 im Gegenzug auf eine Vertragsverletzung berufen kann. Würde ein solcher V freilich im Vertrauen, die erste emptio venditio rückgängig machen zu können, eine zweite über dasselbe Objekt abschließen, und käme es dann vor dem zufälligen Untergang der res vendita - weder zu einer traditio noch zu einem pretium accipere, so hätte sich wohl auch Paulus für die "strenge Consequenz" entschieden, die eine andere, in D. 18.4.21 bloß zitierte Juristenmeinung bei dem vom Spätklassiker gelösten "hard case" vertritt ${ }^{83}$.

M. Pennitz ${ }^{84}$

Innsbruck

82 Vgl. Jhering, Sinn (Fn. 2), 461 f.; 482 f. sowie schon oben bei Fn. 52.

83 S. schon oben bei Fn. 3. Da Pennitz, Periculum (Fn. 17), 111; 112 f.; 115 die "andere" Juristenmeinung mit jener von Paulus identifiziert hat, bietet sich im Übrigen an dieser Stelle gleichfalls die Möglichkeit, einen "Irrthum ... öffentlich zurückzunehmen".

Prof. Dr. Martin Pennitz ist Professor Römisches Recht an der Universität Innsbruck. 